\title{
Aus:
}

Caroline Schmitt, Asta Vonderau (Hg.)

\section{Transnationalität und Öffentlichkeit}

Interdisziplinäre Perspektiven

Juni 20I4, 340 Seiten, kart., 34,99€, ISBN 978-3-8376-2154-9

Wie können transnationale Öffentlichkeiten untersucht werden? Ob in Zusammenhang mit der globalen AIDS-Epidemie, in migrantischen Organisationen, im Umfeld der pentekostalen Kirche oder im Zuge der neuen sozialen Bewegungen: Weltweit entstehen diskursive Arenen und Formen zivilgesellschaftlichen Engagements, deren Handlungsspektrum und politische Wirksamkeit die Grenzen von Nationalstaaten überschreiten und das traditionelle Verständnis von Öffentlichkeit in Bewegung bringen.

Solche empirisch beobachtbaren Knotenpunkte transnationalen Kommunizierens und Handelns werden in diesem Band von einer multidisziplinären Beiträgerschaft als situative und temporäre Öffentlichkeiten betrachtet und analysiert.

Caroline Schmitt (Dipl.-Päd.) ist wissenschaftliche Mitarbeiterin am Institut für Erziehungswissenschaft der Universität Mainz (Sozialpädagogik).

Asta Vonderau (Prof. Dr.) ist Juniorprofessorin für Kulturanthropologie am Institut für Theater-, Film- und empirische Kulturwissenschaft an der Universität Mainz und Senior Researcher am Institut für Sozialanthropologie an der Universität Stockholm.

Weitere Informationen und Bestellung unter:

www.transcript-verlag.de/978-3-8376-2154-9

(C) 2014 transcript Verlag, Bielefeld 


\section{Inhalt}

Öffentlichkeiten in Bewegung

Caroline Schmitt und Asta Vonderau | 7

\section{Praktiken und Bühnen des Öffentlich-Werdens}

Öffentlichkeit unter den Bedingungen urbaner Superdiversität: Überlegungen zum Umgang mit einer Kategorie in den Kulturwissenschaften

Ina Dietzsch | 27

Die Bedeutung von Lokalität für die Teilhabe an multiplen Öffentlichkeiten in einer transnationalen Pfingstkirche

Kristine Krause | 55

Marktplätze der Öffentlichkeit:

Der Aufstand des Informellen gegen die globale Ökonomie Peter Mörtenböck | 8I

\section{Situative Sichtbarkeiten}

Exterritoriale Grenzpolitik als Moment transnationaler Öffentlichkeit

Lena Laube | II3

Geografien der Öffentlichkeit: Transnationale vietnamesische Initiativen und ihre kommunale Steuerung in Berlin Antonie Schmiz | I43 


\section{WisSensVermittLeR UNd MULtiple EngagementS}

No Public? Class Dynamics, the Politics of Extraversion, and the Non-Formation of Political Publics and (Religious) AIDS Activism in Urban Tanzania

Hansjörg Dilger | I75

"Für die Ausübung ihrer zukünftigen Freiheit" - Zur Deutung und Herstellung von Öffentlichkeit in einem Migrantinnenverein Annemarie Duscha $\mid 207$

Nichtregierungsorganisationen als Interessenvertreter und Politikvermittler in einer transnationalen öffentlichkeit Christiane Frantz | 233

Migrant Associations' Double Engagement and the Transnationalisation of Public Spheres Bruno Riccio | 26I

\section{Digitale und translokale Koalitionen}

Öffentlichkeit, Gegenöffentlichkeiten und Medienpartizipation im Zeitalter des Internets

Jeffrey Wimmer | 285

Jugendprotest und transnationale öffentlichkeit Arne Schäfer/Matthias D. Witte | 309

Autorinnen und Autoren | 335 


\title{
Öffentlichkeiten in Bewegung
}

\author{
Caroline Schmitt und Asta Vonderau
}

Ob der Arabische Frühling in Ägypten und anderen nordafrikanischen Ländern, die Proteste im Istanbuler Gezi Park oder die Aktionen der Femen und der Occupy-Bewegung: Ereignisse wie diese gehen um die Welt, weil über sie berichtet wird, vor allem aber, weil sie in anderen gesellschaftlichen Kontexten Initiativen anstiften und damit Orte und Menschen miteinander verbinden. Sie lassen neue Kommunikations- und Handlungsräume entstehen, die als Bühnen grenzüberschreitender Öffentlichkeiten fungieren - Bühnen, die Individuen oder Organisationen eine Präsenz verschaffen, die neue Formen gesellschaftlicher Diskussion und Teilhabe ermöglicht.

Doch nicht nur öffentlichkeitswirksame Aktionen haben das Potenzial, >um die Welt zu gehen $<$ und als transnationale öffentliche Foren zu fungieren. Vielmehr verdeutlichen die im vorliegenden Band versammelten Beiträge die Bandbreite lokaler alltagsweltlicher und institutioneller Kontexte - von pentekostalen Kirchen, informellen Märkten über Jugendproteste und Grenzpolitiken bis hin zu anti-AIDS-Aktionen, Migrantenvereinen ${ }^{\mathrm{I}}$ oder NGOs -, in deren Rahmen sich grenzübergreifende Beziehungen anbahnen können. Unser Anliegen ist es, gerade auf diese weniger sichtbaren, scheinbar marginalen Situationen und Kontexte des sozialen Lebens aufmerksam zu machen, die Öffentlichkeiten darstellen und als solche zu untersuchen sind.

Diese dynamischen, oft temporären und transnational verorteten Formen von Öffentlichkeit sind indes nicht nur als empirische Tatsachen zu

1 | Aus Gründen der Lesbarkeit verwenden wir in unserem Beitrag die männliche Geschlechterform. Frauen sind jedoch selbstverständlich mit inbegriffen. Gleiches gilt für die in diesem Band versammelten Beiträge. 
nehmen, die von einer Forscherin lediglich aufgesucht und beschrieben werden müssen. Sie zeigen auch eine analytische Perspektivenerweiterung an, die in der interdisziplinären Diskussion des letzten Jahrzehnts zu Formen und Wirkungsweisen transnationaler Kommunikations- und Handlungsräume deutlich wurde (vgl. z. B. Castells 2008; Crack 2008; Fraser 2008; Kaelble/Kirsch/Schmidt-Gernig 2002). Diese Diskussion zeichnet sich durch die Bereitschaft und das Interesse aus, Öffentlichkeit als Kategorie neu zu denken. Auch dazu will der vorliegende Band beitragen. Er versammelt Beispiele qualitativer empirischer Forschungen aus sozial- und kulturwissenschaftlichen Disziplinen, die unterschiedliche disziplinäre, forschungspraktische und methodische Vorgehensweisen sowie theoretische Konzeptionalisierungen von Öffentlichkeit präsentieren: Kulturanthropologie und Europäische Ethnologie, Ethnologie, Pädagogik, Geografie, Kommunikations- und Medienwissenschaften, Politikwissenschaft sowie Soziologie. Gemeinsam ist den Beiträgen die Analyse transnationaler Settings, innerhalb derer grenzüberschreitende Öffentlichkeiten identifiziert und untersucht werden. Gerade die Heterogenität der empirischen und theoretischen Zugänge stellt die Besonderheit des Bandes dar, dem es daran gelegen ist, Verbindungen zwischen den unterschiedlichen disziplinären Perspektiven herzustellen und die interdiziplinäre Diskussion zu materiellen Formen, kulturellen Bedeutungen und sozialen Wirkungsweisen von transnationalen Öffentlichkeiten somit in Bewegung zu bringen.

Dem interdisziplinären Ansatz des Bandes entsprechend, legen wir unseren weiteren Überlegungen einen erweiterten Öffentlichkeitsbegriff zu Grunde. Wir verstehen Öffentlichkeit als einen relationalen, zeitlich und räumlich situierten und multipel verorteten Raum verdichteter Handlung und Kommunikation - »a specific historical, political and economic conjuncture in which an issue becomes a problem« (Ong/Collier 2005:I4) ${ }^{2}$. Innerhalb dieses Raums treffen verschiedene Akteure und Interessen aufeinander, um Lösungen für soziale Probleme zu verhandeln. Wichtig ist es für uns im Zuge dieser begrifflichen Öffnung erstens, die in gängigen Konzeptionen von Öffentlichkeit dominierenden Dichotomien von lokal/ global, formell/informell, öffentlich/privat, räumlich/medial aufzuweichen und diese Kategorien quer (als komplexe Relationen statt Dichotomien) zu denken. Zweitens möchten wir den prozessualen Charakter transnationaler

2 | In einem etwas anderem Zusammenhang bei Collier und Ong. 
Öffentlichkeiten betonen und auf deren zeitliche und räumliche Situativität aufmerksam machen. Drittens will der vorliegende Band die Komplexität und Verwobenheit multipler Kollaborationen der in Prozesse der Öffentlichkeitsbildung involvierten Akteure sichtbar machen. Dabei geht es uns weniger um endgültige Definitionen und reduzierende Modelle als vielmehr darum, Komplexitäten aufzuzeigen, die transnationale Öffenlichkeiten erzeugen und ausmachen, und schließlich interessiert uns die Frage, wie solche Komplexitäten analytisch und empirisch gefasst werden können.

\section{Konzeptionelle Erweiterungen}

Einen erweiterten Begriff von Öffentlichkeit zur Grundlage einer interdisziplinären Diskussion zu nehmen, ist aufgrund seiner historisch etablierten Konnotationen und eingespielten disziplinären Verwendungen kein selbstverständliches Unterfangen. Spätestens seit Jürgen Habermas' Strukturwandel der Öffentlichkeit (2013 [1962]) ist Öffentlichkeit ein vor allem in den Politikwissenschaften gebräuchlicher Begriff und eine »epochaltypische Kategorie« (ebd. 2013 (I962): 5I), die eng mit den westlichen Modellen der Demokratie und des Nationalstaates verknüpft und daher klar territorialisiert ist. Die Frage nach der Territorialität von Öffentlichkeit gerät angesichts der Transnationalisierung gesellschaftlicher Prozesse (vgl. z. B. Mau 2007; Pries 2003, 2008; Wimmer/Glick Schiller 2002) ins Zentrum weiterführender Diskussionen, welche die von Habermas identifizierten Aspekte von Öffentlichkeit - ihr kritisches Potential, ihre Normativität, politische Legitimität und Effektivität - über ein nationalstaatliches Territorium hinausführen und aus grenzüberschreitender Perspektive diskutieren (vgl. z. B. Fraser 2008; Nash 2007; Tobler 20I0). Dabei ändert sich vor allem das Verständnis der Teilname an und der Zugehörigkeit zu einer bestimmten Öffentlichkeit, die nicht mehr nur als eine durch nationalstaatliche Bürgerschaft bestimmte verstanden wird. Stattdessen wird die Betroffenheit von Akteuren -ihre »wechselseitige Verstrickung in Strukturen und/oder Institutionen « (Fraser 2008: 3I) - zum Entfaltungsund Inklusionsprinzip von Öffentlichkeiten erhoben. Diese strukturellen und institutionellen Verstrickungen können im Territorium eines Nationalstaates, aber auch grenzüberschreitend, auf verschiedenen räumlichen Ebenen (scales) des gesellschaftlichen Lebens - lokal, urban, regional, national, transnational - entstehen und vielfältige territoriale Ausformungen 
annehmen. Aus einer normativen Perspektive verfügen solche Öffentlichkeiten erst dann über politische Legitimität, wenn »alle von einer politischen Entscheidung potentiell Betroffenen die Chance zur gleichberechtigten Teilnahme an den informellen Prozessen der Meinungsbildung« (Fraser 2008: 30) erlangen können. Dieser Sichtweise folgend, muss die von den Betroffenen gebildete Meinung von einem oder mehreren Nationalstaaten und Entscheidungsträgern aufgegriffen und in geltendes Recht und administrative Macht übersetzt werden. Die Schaffung neuer transnationaler Gewalten zeigt sich, wie Nancy Fraser betont, als unbedingt notwendig, um diese normative Funktion solcher Öffentlichkeiten realisieren zu können. (vgl. ebd. 2008: 3off.) Dass die empirisch beobachtbaren Formierungsprozesse von transnationalen Öffentlichkeiten ebenso komplex wie konfliktreich sind und in den meisten Fällen nur zum Teil dem normativen Modell einer legitimen Öffentlichkeit entsprechen, ist offensichtlich. Kate Nash (2007) beispielsweise gibt kritisch zu bedenken, dass die in normativen Öffentlichkeitsmodellen berücksichtigten Strukturen und Wege der Meinungsäußerung vielfach von politischen Eliten geprägt sind und marginalisierte Gruppen von Meinungsbildungsprozessen ausschließen. Entsprechend bleiben diese Gruppen in den auf staatliche Strukturen und Akteure fokussierten Öffentlichkeitskonzeptionen meist unberücksichtigt. Auch Oelsen (2005) betont, dass die Meinungsäußerungen marginalisierter Gruppen ebenfalls als Öffentlichkeiten betrachtet werden sollten, was zu einer Pluralisierung des Öffentlichkeitsverständnisses führt, insofern statt nur einer dominanten Öffentlichkeit die Existenz von weiteren, auch widersprüchlichen oder konträren Gegenöffentlichkeiten anerkannt wird (vgl. Oelsen 2005: 435).

Es lässt sich also festhalten, dass die interdisziplinäre Diskussion der letzten Jahre von einem steigenden Bewusstein darüber geprägt ist, dass angesichts der zunehmenden Mobilitäten von Individuen, Politiken, Organisationen und der damit verbundenen »Verschiebung der Problemlagen auf die transnationale Ebene« (Kaelble/Kirsch/Schmidt-Gernig 2002, S. 7) eine Pluralisierung und Erweiterung des Öffentlichkeitsverständnisses relevant wird. Denn diese Prozesse transnationaler Kommunikation lassen sich nicht mit dem auf nationalstaatliche Strukturen bezogenen Öffentlichkeitsverständnis allein erfassen. Vor allem Kommunikations- und medienwissenschaftliche Konzeptionen haben zu dieser Erweiterung beigetragen und den Begriff der Öffentlichkeit mit weiteren Konnotationen versehen. Öffentlichkeit wird in diesen Disziplinen vor allem als Kom- 
munikationsfluss und medialer Prozess der Meinungsbildung analysiert (vgl. z. B. Brüggemann et al. 2009; Doerr 20I0; Koopmans 2004; Wallner/ Adolf 20II; Wimmer 2007). Untersuchungen von globalen medialen Vernetzungen geben nicht zuletzt Nahrung für wissenschaftliche und alltagsweltliche Visionen einer gänzlich entgrenzten Kommunikation, die sich der Idealvorstellung einer von räumlichen sowie sozialen Zwängen und Ausgrenzungen >freien< Öffentlichkeit annähert. Doch auch solche Visionen werden durch das Erkennen der den medialen Informationsflüssen inhärenten räumlichen und sozialen Bezüge und Spannungen relativiert (vgl. Wimmer in diesem Band). Im Rahmen dieser disziplinären Diskussion wird zugleich festgestellt, dass die zunehmende Verbreitung des Öffentlichkeitsbegriffs, insbesondere in Bezug auf transnationale gesellschaftliche Kontexte, weniger zu seiner definitorischen Klarheit als vielmehr dazu beiträgt, Öffentlichkeitskonzepte zu überdenken (Wimmer 2007: 2I, Brüggemann et.al. 2009). Zusammenfassend kann festgehalten werden, dass sowohl eine Verengung des Öffentlichkeitsverständnisses auf nationalstaatliche Territorien als auch seine Ausweitung auf grenzenlose globale Informationsströme im Laufe der letzten Jahre hinterfragt und kritisch reflektiert wurde. Im Rahmen dieser Auseinandersetzung sind vielfältige grenzüberschreitende Komplexitäten der Öffentlichkeitsbildung ins Zentrum des wissenschaftlichen Interesses gerückt, die weder völlig entgrenzt, noch eindimensional (ausschließlich lokal oder national) verräumlicht sind. Die empirische und analytische Erfassung solcher Komplexitäten stellt eine Herausforderung für die aktuelle interdisziplinäre Diskussion dar. In diesem Zusammenhang wurde bereits auf einen Mangel an akteurs- und praxiszentrierten, empirischen Studien hingewiesen, welche die zeitlichen und räumlichen Ausformungen und Effekte transnationaler Öffentlichkeiten am konkreten Fall analysieren (vgl. z. B. Nash 2008 sowie Dietzsch in diesem Band).

\section{Praktiken und Bühnen des Öffentlich-Werdens}

Angesichts der oben erwähnten Forschungsdefizite liegt es nahe, Öffentlichkeiten nicht ausschließlich als politische Projekte und diskursiv-kommunikative Beziehungen zu betrachten, sondern als zeitlich wie räumlich situierte, körperlich markierte gelebte Erfahrungen und Alltagspraktiken. Wir plädieren mit diesem Band daher für eine intensivere Erforschung von 
Praktiken des Öffentlich-Seins, -Werdens, und -Machens in transnationalen Kontexten, im Sinne eines doing publics (vgl. Dietsch in diesem Band), was die auf gesellschaftliche Strukturen oder Kommunikationsströme fokussierten Forschungsperspektiven produktiv ergänzen und erweitern kann.

Ansätze, die zumindest am Rande auf Akteursperspektiven und Alltagspraktiken des Öffentlich-Seins und -Machens eingehen, gibt es in geringer Zahl bereits. So weisen Gerhards und Neidhardt (I990, 20ff.) auf die Notwendigkeit hin, neben der massenmedialen Kommunikation und dem kollektiven Protest (der Ebene öffentlicher Veranstaltungen) auch die alltägliche Kommunation auf der Straße (encounter) zu berücksichtigen, um bestimmen zu können, »was Öffentlichkeit ist, welche Funktionen ihr in modernen Gesellschaften zukommen, welche Strukturen sie aufweist und welche Forschungsfragen sich daraus ergeben« (Gerhards/Neidhardt I990: 6). Aus heutiger Sicht ließen sich auch viele weitere, miteinander verschränkte und überlappende Ebenen und Skalen der Öffentlichkeitsbildung beschreiben - beispielsweise neue Kommunikationskanäle wie Blogs oder Online-Plattformen sowie Aktionen der Neuen Sozialen Bewegungen -, in deren Rahmen Praktiken des Öffentlich-Seins und -Machens beobachtet und analysiert werden können. Praktiken sind in diesem Zusammenhang als grenzüberschreitend in Bezug auf staatliche Territorien, aber auch in Bezug auf den physischen und virtuellen Raum zu verstehen. Öffentliche Aushandlungen erfolgen nicht ausschließlich »au trottoir«, sondern können ebenso virtuell ablaufen. Öffentliche Aktionen können durch verschiedene geografische und zeitliche Räume hindurch organisiert sein; man denke etwa an den Tanz-Flashmob vom 21. September 2013 anlässlich des Internationalen Tages des Friedens, ${ }^{3}$ an dem Personen in I80 verschiedenen Städten weltweit teilnahmen. Insgesamt eröffnet eine praxeologische Vorgehensweise in Verbindung mit strukturell ausgerichteten und auf Kommunikationsströme fokussierten Forschungsperspektiven (vgl. Wimmer in diesem Band) die Möglichkeit, transnationale Ausformungen diverser Öffentlichkeiten durch verschiedene lokale Kontexte hindurch zu verfolgen, ohne dabei ihre räumlichen Bezüge und die ihnen inhärenten Widersprüche und Konflikte aus den Augen zu verlieren (vgl. Krause in diesem Band). Ein solches Vorgehen berücksichtigt nicht nur die neuen Kommunikations- und Handlungsformen, die im Rah-

3 | Homepage zum internationalen Flashmob anlässlich des Internationalen Tages des Friedens: http://www.izfm.org/. 
men transnationaler Öffentlichkeiten möglich werden, sondern auch die Grenzen solcher Öffentlichkeiten (vgl. Dietsch, Dilger und Mörtenböck in diesem Band) und die ständigen Verschiebungen der Interessen und Engagements der in diesen Öffentlichkeiten involvierten und von ihnen betroffenen Akteure.

Werden Öffentlichkeiten von den alltäglichen Praktikenen des Öffentlich-Seins und -Machens aus gedacht, können die identitätsstiftenden Bedeutungen der im Prozess der Öffentlichkeitsbildung entstehenden Koalitionen, Beziehungen und Engagements für indivuelle Lebensentwürfe und kollektive Handlungsmotive in einem lebensweltlichen Sinne erfasst werden. So zeigen Schäfer und Witte in ihrem Beitrag für dieses Buch, wie sich Jugendliche durch das Engagement in transnationalen Protestbegweungen als Agenten begreifen und Selbstwirksamkeit erfahren. Auch Kaelble et al. (2002) sowie Schmiz (in diesem Band) erörtern, inwiefern mit der Teilnahme an transnationalen Öffentlichkeiten eine Formation individueller und kollektiver Identitäten einhergeht, die nicht auf dauerhafter Zugehörigkeit zu einer territorial oder auf andere Weise eindeutig definierten Gemeinschaft basieren, sondern als hybrid und flexibel charakterisiert werden können. Aus einer praxeologischen Perspektive betrachtet sind Öffentlichkeiten also weniger feste räumliche oder soziale Größen, sondern vielmehr komplexe, sich in ständiger Bewegung befindende überlappende Zonen gesellschaftlicher Aushandlungen - Öffentlichkeiten in the making. Diese Aushandlungszonen sind zum einen multipel verortet; sie können die lokalen, regionalen, nationalen räumlichen und administrativen Grenzen, aber auch scheinbar getrennte Bereiche gesellschaftlichen Lebens (das Private und das Öffentliche, das Formelle und das Informelle) überschreiten und auf diverse Weisen miteinander verbinden. Zum anderen geben sie Individuen und Kollektiven selbst die Möglichkeit der multiplen Indentifizierung und Selbsverortung. Entsprechend betrachten die Autoren dieses Bandes transnationale Öffentlichkeiten als Aushandlungszonen, in deren Rahmen neue Formen gesellschaftlicher Teilhabe, Meinungs- und Identitätsbildung, aber auch neue Konflikte und Ausgrenzungen entstehen.

Eine solche, auf die Praktiken der Herstellung von Öffentlichkeiten sowie auf kulturelle Deutungsprozesse gerichtete Perspektive entwickelt Ina Dietzsch in ihrem Beitrag »Öffentlichkeit unter den Bedingungen urbaner Superdiversität: Überlegungen zum Umgang mit einer Kategorie in den Kulturwissenschaften«. Im Rahmen einer Baseler Fallstudie zeigt sie, 
wie an einem Ort Öffentlichkeiten unterschiedlicher Reichweite und verschiedene Öffentlichkeitsverständnisse zusammen kommen. Die Autorin hebt dabei die Rolle der Medien als »scaling devises« hervor, die sich für das Entstehen und die Struktur der Öffentlichkeiten als besonders wichtig erweisen. Medien tragen auch dazu bei, dass transnationale Prozesse lokal als eine besondere Form des gesellschaftlichen Zusammenlebens als »nahe Diversität« (Koch 20I2:I20) - erfahrbar werden. Kristine Krause untersucht in ihrem Beitrag »Die Bedeutung von Lokalität für die Teilhabe an multiplen Öffentlichkeiten in einer transnationalen Pfingstkirche« Grenzziehungs- und Inkorporierungspraktiken im Kontext der grenzüberschreitenden pentekostalen Öffentlichkeit, die sie als Konstellation räumlich, situativ und temporär gebundener und zugleich durch globale Machtgeometrien geprägter diskursiver Arenen versteht. Am Beispiel einer von Zuwanderern aus Ghana in Deutschland gegründeten Pfingstkirche beschreibt Krause, wie sich die Kirche je nach Referenzrahmen als deutsch, afrikanisch, migrantisch, international oder global präsentiert und entsprechend wahrgenommen wird. Die Autorin betont, dass erst die Berücksichtigung der strategischen Bedeutung von Lokalitäten sowie die lokal beobachtbaren Grenzziehungs- und Zuschreibungspraktiken eine genaue Beschreibung und Konzeptionalisierung transnationaler Öffentlichkeiten ermöglicht. Peter Mörtenböck betrachtet in seinem Beritrag »Marktplätze der Öffentlichkeit. Der Aufstand des Informellen gegen die globale Ökonomie« informelle Märkte als Orte, an denen transnationale Öffentlichkeiten von unten entstehen. Er zeigt, wie Freiluftmärkte Einkommens- und Teilhabemöglichkeiten für Gruppen und Individuen eröffnen, die von den formellen neoliberalen Märkten nicht profitieren können. Mörtenböck betrachtet die ökonomisch wie politisch begründeten Beziehungen und Kollaborationen der auf den informellen Märkten aktiven Akteure als »Bruch mit der vorhandenen Definitionsmacht« und als einen Kampf um die Legitimität alternativer »Arrangements von gesellschaftlichem Austausch, deren Gestalt, Streuung und Verteilung«. Der überindividuelle Charakter von Praktiken informellen Handels und der gesellschaftlichen Teilhabe lässt Informalität als Ausdruck von kollektiver Selbstbestimmung und politischem Widerstand erscheinen. 


\section{Situative Sichtbarkeiten}

Transnationale Zonen gesellschaftlicher Aushandlung und Auseinandersetzung verfügen, wie die hier versammelten Beiträge zeigen, über das Potenzial, zu Generatoren neuer Partizipations- und Teilhabeformen zu werden, die jedoch nicht notwendigerweise dauerhaft sind. Diese Zonen sind in der Regel nur temporär und überdies im ständigen Wandel begriffen. Wie Jeffrey Wimmer in seinem Beitrag anmerkt, erweisen sich selbst die durch klassische Institutionen, politische Eliten und etablierte Verfahren der Meinungsbildung strukturierten und scheinbar festen Kommunikationsräume bei genauerem Hinsehen als instabil. Sie zerfallen in segmentierte Öffentlichkeiten unterschiedlicher Komplexität und Reichweite. Diese Segmentierung deckt die sehr unterschiedlichen Kommunikationslogiken und widersprüchlichen Prozesse der Öffentlichkeitsbildung auf, ebenso wie die immer wieder neuen Konstellationen der an diesem Prozess beteiligten und von ihnen betroffenen Akteure. Auf ähnliche Weise schildert Kristine Krause, wie transnationale Öffentlichkeiten durch die unterschiedlichen Positionierungen ihrer Akteure und aufgrund diverser lokaler Voraussetzungen verschiedene Formen und Reichweiten erlangen, und wie sie durch globale Machtkonstellationen mitgestaltet werden.

Aufgrund ihres temporären Charakters verstehen wir transnationale Öffentlichkeiten, Peter Mörtenböck folgend, weniger als feste Institutionen denn als bewegliche politische Ökologien und Projekte, die an unterschiedliche Erwartungen und Interessen situativ anknüpfen. Die Effekte solcher Projekte sind nicht als ausschließlich geradlinig oder planbar zu verstehen, sondern vielmehr von Zufälligkeiten, Improvisationen und unerwarteten Wenden geprägt. Es bedarf also einer jeweils spezifischen soziomateriellen Konstellation, damit diese Projekte ihre öffentlichkeitsgenerierende Funktion überhaupt erlangen und zu »Öffentlichkeitsmaschinen« (vgl. Mörtenböck in diesem Band) werden können.

Ein Beispiel solcher Situativität und Temporalität gibt Lena Laube in ihrem Beitrag »Exterritoriale Grenzpolitik als Moment transnationaler Öffentlichkeit«. Sie schildert eine Begegnung zwischen den Techniken der europäischen Grenzkontrolle einerseits (die heute nicht mehr ausschließlich an den nationalstaatlichen Grenzen selbst, sondern an verschiedenen Orten lokalisiert sind), und den zu konktrollierenden migrierenden Individuen anderseits. In dieser durch ungleiche Machtverhätlnisse geprägten Situation sieht die Autorin dennoch Potenzial für das Entstehen einer tempo- 
rären transnationalen Zone gesellschaftlicher Auseinandersetzung - einen potentiellen »Moment« transnationaler Öffentlichkeit -, der dann eintritt, wenn die Grenze ihre Regel offenbart, wenn andere politische Akteure (beispielsweise NGOs) aktiv werden und wenn sie eine kritische, über die jeweiligen Nationalstaaten hinausgehende Diskussion und Aktion initiieren.

Die genaue Betrachtung solch scheinbar marginaler >Öffentlichkeitsmomente < verdeutlicht nicht nur die jeweiligen strukturellen Voraussetzungen und die in dem jeweiligen Kontext einflussreichen politischen Diskurse, sondern auch die Machtgefälle und die ungleich verteilten Handlungsoptionen und Möglichkeiten von Meinungsäußerung in ihrer alltagsweltlichen räumlichen und körperlichen Ausprägung. Ohne Zweifel sind die offiziell anerkannten institutionalisierten Akteure - wie beispielsweise NGOs und insbesondere Medien - oft die einflussreichsten Gestalter öffentlicher Aushandlungen. Sie können die Reichweite sowie Sichtbarkeit eines bestimmten Problems und der sich darum gruppierenden Öffentlichkeit entscheidend formen. Doch zeigen sich bei Betrachtungen kurzzeitiger und momentartiger Öffentlichkeitsbildungen durchaus auch andere, weniger etablierte, informell vorgehende Akteure, welche Öffentlichkeiten mitprägen und die dominanten Sichtbarkeits- und Wahrheitsregimes stören oder hinterfragen können.

Auf solche situativ sichtbaren Akteure und ihre Strategien des Öffentlich-Werdens konzentriert sich Antonie Schmiz in ihrem Beitrag »Geografien der Öffentlichkeit: Transnationale vietnamesische Initiativen und ihre kommunale Steuerung in Berlin «. Am Beispiel von Demonstrationen vietnamesischer Migranten und am Fall eines asiatischen Großhandelszentrums in Berlin zeigt Schmiz, wie diese urbanen Kontexte temporäre transnationale Öffentlichkeiten generieren, deren Dauer und Reichweite von der sich wandelnden Konstellation der beteiligten Akteure abhängen und durch sie mitbestimmt werden. Es sind vor allem kommunale Akteure (die Stadt Berlin und die involvierten Bezirke), denen in diesem Zusammenhang Gestaltungsmacht und Einfluss darauf zukommen, welche Form von »publicity« den vietnamesischen Demonstranten und Händlern des Großhandelszentrums ermöglicht wird.

Zusammenfassend stellt eine stärkere Berücksichtigung der zeitlichen Dimensionen und des situativen Charakters von Öffentlichkeitsbildung eine besonders ergiebige Diskussionsrichtung dar, welche die räumlichen Ausprägungen transnationaler Öffentlichkeiten mit den zeitlichen Verschiebungen der diesen Öffentlichkeiten inhärenten Interessen, Bedürf- 
nissen und Engagements zusammen zu bringen vermag. Um diese Bezüge herstellen zu können, erscheint uns eine Ergänzung der von Gerhards und Neidhardt (I990: 6) vorgeschlagenen Frage danach notwendig, was Öffentlichkeit ist und welche Funktionen sie in einer Gesellschaft erfüllt. Berücksichtigt werden muss auch und vor allem die Frage, wann, das heißt unter welchen historischen und sozialen Voraussetzungen transnationale Handlungs- und Kommunikationsräume entstehen, wie sie sich im Sinne einer zeitlichen und räumlichen Ausprägung und Reichweite konstituieren, und für wen sie Sichtbarkeit ermöglichen oder als Bühnen des Öffenlich-Machens und -Werdens erfahrbar sind.

\section{Multiple Engagements und Koalitionen}

Transnationale Öffentlichkeiten weisen polymorphe Geografien auf, die nicht nur verschiedene lokale Kontexte umfassen, sondern auch virtuelle und physische Räume verbinden. Da die Reichweite und Dauer solcher Öffentlichkeiten sehr unterschiedlich sein können und in ständigem Wandel begriffen sind, ist die Teilnahme ihrer Akteure weniger als dauerhafte Zugehörigkeit oder längerfristiges, durchgeplantes Handeln zu verstehen, sondern als ein multiples und flexibles Engagement. Sind es nicht mehr nationale oder andere feste Zugehörigkeiten, die das organisierende Prinzip solcher Öffentlichkeiten ausmachen, sondern die sich wandelnden Interessen und diversen Grade von Betroffenheit, dann werden mehrfache überlappende Engagements und gleichzeitige Zugehörigkeiten der an transnationalen Öffentlichkeiten beteiligten Akteure nicht nur möglich, sondern sogar üblich und alltäglich. Die hier versammelten Beiträge schildern eine ganze Reihe solcher mehrfachen Bezüge und Engagements.

Hansjörg Dilger zeigt in seinem Beitrag »No Public? Class dynamics, the Politics of Extraversion, and the Non-Formation of Political Publics and (Religious) AIDS Activism in Urban Tanzania«, wie die Anti-AIDS/ HIV Aktionen in zwei unterschiedlichen Kontexten - den USA und Tansania - von unterschiedlichen (sowohl lokal als auch grenzüberschreitend handelnden) Akteuren mit ihren jeweiligen heterogenen Interessen aufgenommen und realisiert werden. Dabei beschreibt Dilger vor allem Widersprüche und unterschiedliche Entwicklungen dieser Projekte, die im Prozess der transnationalen Wissens- und Erfahrungsvermittlung (etwa zur AIDS-Prävention oder zu den möglichen Protestformen) und ange- 
sichts ihrer unterschiedlichen lokalen soziokulturellen Voraussetzungen entstehen und die Herausbildung von Öffentlichkeiten fördern (oder auch hemmen). In »>Für die Ausübung ihrer zukünftigen Freiheit<- Zur Deutung und Herstellung von Öffentlichkeit in einem Migrantinnenverein« analysiert Annemarie Duscha, wie sich eine brasilianische Migrantinnenorganisation in Deutschland um die Herstellung einer für die Migrantinnen zugänglichen Öffentlichkeit engagiert, in dem sie sowohl nationale als auch transnationale Koalitionen initiiert und diese in Verbindung bringt. Durch ihr multiples Engagement generiert die Organisation »ein gemeinsames kollektives Wissen über das Leben als Migrant in Europa.« Ein solches Engagement in Sachen Wissensvermittlung versteht die Autorin als Akt der Bildung, der den Handlungsspielraum von Migrantinnen erweitert. In vergleichbarer Weise beschreibt Christiane Frantz in ihrem Beitrag »Nichtregierungsorganisationen als Interessenvertreter und Politikvermittler in einer transnationalen Öffentlichkeit« Aktivitäten und Präsenzen der transnational verorteten NGO care. Die Autorin zeigt, wie NGOs durch ihre gleichzeitigen lokalen und transnationalen Engagements zu einflussreichen Akteuren in einem medial vermittelten Prozess der Bildung politischer Öffentlichkeiten werden. Auch Bruno Riccio stellt in seinem Beitrag »Migrant Associations' Double Engagement and the Transnationalisation of Public Spheres« anhand von Fallbeispielen senegalesischer Migrantenorganisationen in Italien fest, dass diese Organisationen zur Transnationalisierung von Öffentlichkeit beitragen können, insofern sie mit ihren multiplen Engagements sowohl nationale als auch binationale Entwicklungskonzepte erweitern und in mehreren gesellschaftlichen Kontexten gleichzeitig politisch wirksam sind. Diese grenzüberschreitenden und einander überlappenden Aktivitäten fasst Riccio unter dem Begriff des »Co-Development« zusammen.

Die mehrfach engagierten und in multiplen temporären Koalitionen involvierten Akteure bedienen sich - dies machen alle in diesem Band versammelten Beiträge deutlich - einer Kombination aus digitalen und lokalen Kommunikations- und Handlungswege. Sie verfügen über einen »doppelten« (oder gar mehrfachen) Raumbezug (vgl. Schäfer/Witte in diesem Band), den sie als Ressource für die Schaffung neuer Kommunikationsformen, Handlungsmöglichkeiten sowie individueller und kollektiver Identitäten nutzen. Jeffrey Wimmer erläutert in seinem Beitrag »Öffentlichkeit, Gegenöffentlichkeiten und Medienpartizipation im Zeitalter des Internets«, wie ein solcher doppelter Raumbezug die subjektiven und struktu- 
rellen Dimensionen von Öffentlichkeit verändert und Partizipations- sowie Koalitionsmöglichkeiten von Akteuren erweitert. Zugleich weist Wimmer darauf hin, dass die neuen Kommunikationstechnologien diversen »power laws « unterliegen und nicht unendliche Engagements und Beziehungen ermöglichen. Sie können überdies reaktionäre Koalitionen befördern und führen somit nicht zwangsweise zu mehr Demokratie. Am Beispiel des G8Gipfels in Heiligendamm im Jahr 2007 als auch des Arabischen Frühlings analysieren Arne Schäfer und Matthias D. Witte in ihrem Beitrag »Jugendprotest und transnationale Öffentlichkeit« das gleichzeitige und ineinandergreifende Engagement protestierender Jugendlicher in lokalen und virtuellen Räumen. Sie beschreiben, wie der »Widerstand im Netz« (Winter 20I0) zu Aktionen und Aktivitäten auf der Straße, in Vereinen, Organisationen und anderen nicht-virtuellen Mobilisierungskontexten führt, die sich wiederum in Formen virtuellen Protestes auf Facebook, Blogs und YouTube fortentwickeln und weitere Aktionen nach sich ziehen. Solche »doppelten Raumbezüge« ermöglichen eine fluide »civic agency«, welche die Jugendlichen trotz der politischen, ideologischen und religiösen Unterschiede in einer transnationalen »Koalition auf Zeit « für die Verhandlung und Lösung eines bestimmten Problems verbindet und ihnen Selbstwirksamkeitserfahrungen ermöglicht. Diese miteinander verwobenen, mitunter widersprüchlichen räumlich gebundenen und virtuellen Engagements nachzuverfolgen ermöglicht es zu verstehen, wie die in einem gesellschaftlichen Kontext teilweise unsichtbaren Akteure ihre Agencies in verschiedenen Räumen und durch multipel verortete Koalitionen und Netzwerke akkumulieren, wie sie sichtbar werden und (temporär) an Einfluss gewinnen und/oder ihn wieder verlieren. Die in diesem Prozess entstehenden »Projekte« einer transnationalen Öffentlichkeitsbildung werden durch die stete Fluktuation der beteiligten Akteure und Interessen dabei nicht notwendigerweise unterbrochen, sondern können von anderen Gruppen und Individuen aufgegriffen und weitergeführt werden. Ferner ist für sie charakteristisch, dass die beteiligten Akteure selbst nicht notwendigerweise das gleiche Verständnis von Öffentlichkeit haben müssen, sondern sich in einer kontinuierlichen Verhandlung darüber befinden. 


\section{Following the Public Spheres}

In der Gesamtschau der hier versammelten Beiträge wird deutlich, dass alle ihre Autoren die zunehmende Präsenz und Bedeutung transnationaler Öffentlichkeiten mit ihrer Beobachtung einer Verquickung von Lokalität, Nationalität und Transnationalität (sowie von Virtualität und Territorialität) verbinden. Diese Beobachtung unterfüttert die These, derzufolge transnationale Prozesse nicht zu einem Bedeutungsverlust des Lokalen oder des Nationalstaats führen. Vielmehr können räumliche, institutionelle und alltagsweltliche Ebenen (Scales) im Zuge der Öffentlichkeitsbildung gleichermaßen relevant werden und zusammenwirken.

Aus eben diesem Grund werden diese Ebenen hier nicht dualistisch betrachtet, sondern vielmehr in Bezug zueinander gesetzt. Neben neuen Kommunikations-, Inklusions- und Teilhabeformen analysieren die Autoren dieses Sammelbandes Aushandlungen, Machtkonstellationen, Spannungen, Widersprüche und Ausgrenzungen, die transnationalen Prozessen der Öffentlichkeitsbildung gleichermaßen inhärent sind. Solche Spannungen zeigen sich als bestimmend dafür, inwiefern agentative und/ oder nicht-agentative soziale Strukturen entstehen, die bestimmten Akteuren Handlungsmächtigkeit und öffentlichen Artikulation ihrer Belange ermöglichen, sie für andere Gruppen aber begrenzen (vgl. Scherr 20I2: IO3). Die Beiträge zeigen zugleich, wie neben lokalen strukturellen Bedingungen auch Individuen und Kollektive durch Praktiken und Strategien des Öffentlich-Machens und -Werdens selbst aktiv an der Schaffung agentativer Kontexte und ihrer Grenzen mitwirken. Neben den offiziell geplanten, politisch legitimierten und ökonomisch wie institutionell gefestigten Öffentlichkeitsformen werden hierbei Improvisationen, Imaginationen und unerwartete Entwicklungen berücksichtigt. Stabilität und Lokalität zeigen sich in diesem Zusammenhang als nicht unwichtige, jedoch besonders fragile Größen, deren Herstellung und Erhalt nach kontinuierlicher Verhandlung und Vernetzung verlangen.

Versteht man (transnationale) Öffentlichkeit wie von uns vorgeschlagen als Praxis, so rücken die (nicht-)agentative Rahmenbedingungen als auch Praktiken von Akteuren gleichermaßen in den Blick. So betrachtet erscheint transnationale Öffentlichkeit als eine historisch gewachsene, sozial multipel verortete, im Wandel begriffene, in alltäglichen Praktiken verkörperte sozio-materielle Formation. Deren konkrete Ausformungen, soziale Funktionen und kulturelle Bedeutungen sind im Sinne eines following the 
public (vgl. Marcus i995) zu untersuchen - durch das Nachverfolgen der relevanten Akteure, Sichtbarkeitsdynamiken, Kommunikationsströme, Deutungsprozesse und der Praktiken des Öffentlich-Machens und -Werdens. Aus einem solchen offenen empirischen und analytischen Zugang ergibt sich weniger eine eindeutige Definition von Öffentlichkeit, als vielmehr die Möglichkeit, (transnationale) Öffentlichkeiten in ihrer Komplexität, Kontextualität und Prozessualität neu zu denken.

\section{LITERATURVERZEICHNIS}

Brüggemann, Michael/Hepp, Andreas/Kleinen-von Königslow, Katharina/ Wessler, Hartmut (2009): »Transnationale Öffentlichkeit in Europa: Forschungsstand und Perspektiven«, in: Publizistik 54, S. 39I-4I4.

Castells, Manuel (2008): »The New Public Sphere: Global Civil Society, Communication Networks, and Global Governance«, in: The Annals of the American Academy of Political and Social Science 6r6 (I). S. 78-93.

Crack, Angela M. (2008): Global Communication and Transnational Public Spheres, New York: Palgrave Macmillan.

Collier, Stephen J./Ong, Aihwa (2005): »Global Assemblages, Anthropological Problems«, in: Dies. (Hg.), Global Assemblages: Technology, Politics, and Ethics as Anthropological Problems, Malden: Blackwell, S. 3-2I.

Doerr, Nicole (2010): »Politicizing Precarity, Producing Visual Dialogues on Migration: Transnational Public Spaces in Social Movements«, in: Forum Qualitative Sozialforschung/Forum Qualitative Social Research II (2), Art. 30.

Fraser, Nancy (2008): »Die Transnationalisierung der Öffentlichkeit. Legitimität und Effektivität der öffentlichen Meinung in einer postwestfälischen Welt«, in: Johanna Dorer/Brigitte Geiger/Regina Köpl (Hg.), Medien - Politik - Geschlecht. Feministische Befunde zur politischen Kommunikationsforschung. Wiesbaden: VS Verlag für Sozialwissenschaften, S. I8-34.

Gerhards, Jürgen/Neidhardt, Friedhelm (I990): Strukturen und Funktionen moderner Öffentlichkeit. Fragestellungen und Ansätze (=Veröffentlichungsreihe der Abteilung Öffentlichkeit und soziale Bewegung des Forschungsschwerpunkts Sozialer Wandel, Institutionen und Vermittlungsprozesse des Wissenschaftszentrums Berlin für Sozialforschung), http://www.polsoz.fu-berlin.de/soziologie/arbeitsbereiche/ 
makrosoziologie/mitarbeiter/lehrstuhlinhaber/dateien/GerhardsNeidhardt-I990.pdf, eingesehen am I2.I2.2013.

Habermas, Jürgen (132013 [1962]): Strukturwandel der Öffentlichkeit, Frankfurt a.M.: Suhrkamp.

Kaelble, Hartmut/Kirsch, Martin/Schmidt-Gernig, Alexander (2002): Zur Entwicklung transnationaler Öffentlichkeiten und Identitäten im 20. Jahrhundert«, in: Dies. (Hg.), Transnationale Öffentlichkeiten und Identitäten im 20. Jahrhundert. Eine Einleitung, Frankfurt a.M.: Campus, S. 7-33.

Koch, Gertraud (20I2): »Distanzieren als Schlüsselkompetenz - eine sozialanthropologische Perspektive auf nahe Diversitäten«, in: Stephan A. Jansen/Nico Stehr/Eckhard Schröter (Hg.), Positive Distanz? Multidisziplinäre Annäherungen an den wahren Abstand und das Abstandwahren in Theorie und Praxis, Wiesbaden: VS Verlag für Sozialwissenschaften, S. II9-І27.

Koopmans, Ruud (2004): »Movements and Media: Selection Processes and Evolutionary Dynamics in the Public Sphere«, in: Theory and Society 33, S. 367-39I.

Marcus, George E. (1995): »Ethnography in/of the World System. The Emergence of Multi-Sited Ethnography«, in: Annual Review of Anthropology 24, S. 95-II7.

Mau, Steffen (2007): Transnationale Vergesellschaftung. Die Entgrenzung sozialer Lebenswelten, Frankfurt a.M./New York: Campus.

Nash, Kate (2007): »Transnationalizing the Public Sphere: Critique and Critical Possibilities«, in: Theory, Culture \& Society 24 (4), S. 53-57.

Oelsen, Thomas (2005): »Transnational Publics: New Spaces od Social Movement Activism and the Problem of Global Long-Sightedness «, in: Current Sociology 53 (3), S. 419-440.

Pries, Ludger (2008): Die Transnationalisierung der sozialen Welt, Frankfurt a.M.: Suhrkamp.

Pries, Ludger (2003): »Transnationalismus, Migration und Inkorporation. Herausforderungen an Raum- und Sozialwissenschaften «, in: geographische revue 5 (2), S. 23-39.

Scherr, Albert (2012): »Soziale Bedingungen von Agency. Soziologische Eingrenzungen einer sozialtheoretisch nicht auflösbaren Paradoxie«, in: Stephanie Bethmann/Cornelia Helfferich/Heiko Hoffmann/Debora Niermann (Hg.), Agency. Qualitative Rekonstruktionen und gesell- 
schaftstheoretische Bezüge von Handlungsmächtigkeit, Weinheim/ Basel: Beltz Juventa, S. 99-I2I.

Tobler, Stefan (20I0): Transnationalisierung nationaler Öffentlichkeit. Konfliktinduzierte Kommunikationsverdichtungen und kollektive Identitätsbildung in Europa, Wiesbaden: VS Verlag für Sozialwissenschaften. Wimmer, Andreas/Glick Schiller, Nina (2002): »Methodological Nationalism and Beyond: Nation-State Building, Migration, and the Social Sciences«, in: Global Networks 2 (4), S. 30I-334.

Wallner, Cornelia/Adolf, Marian (20II): »Zur Erklärungskraft von Öffentlichkeitstheorien für Kommunikationsinnovationen. Eine Metastudie zu klassischen Öffentlichkeitstheorien«, in: Jens Wolling/Andreas Will/ Christina Schumann (Hg.), Medieninnovationen. Wie Medienentwicklungen die Kommunikation in der Gesellschaft verändern, Konstanz: UVK, S. 409-425.

Wimmer, Jeffrey (2007): (Gegen-)Öffentlichkeit in der Mediengesellschaft. Analyse eines medialen Spannungsverhältnisses, Wiesbaden: VS Verlag für Sozialwissenschaften.

Winter, Rainer (20I0): Widerstand im Netz. Zur Herausbildung einer transnationalen Öffentlichkeit durch netzbasierte Kommunikation, Bielefeld: transcript.

\section{Websites}

Homepage zum internationalen Flashmob anlässlich des Internationalen Tages des Friedens, http://www.izfm.org, eingesehen am 02.01.20I4. 